\title{
Research Paper: Effects of age and walking speeds on vertical ground reaction force in younger and older adults
}

\author{
Maryam Rastegar ${ }^{1}$, *Seyyed Hosein Hoseini ${ }^{2}$, Mohamad Hosein Naser Melli ${ }^{1}$, Morteza Taffah ${ }^{3}$
}

1. Department of Physical Education and Sport Sciences, Faculty of Physical Education and Sport Sciences, Karaj Branch, Islamic Azad University, Karaj, Iran. 2. Department of Physical Education and Sport Sciences, Faculty of Physical Education, University of Guilan, Rasht, Iran.

3. Department of Sport injuries and Corrective Exercises, Faculty of Physical Education, University of Guilan, Rasht, Iran

Received: 20 Jun. 2016 Accepted: 11 Oct. 2016

Keywords: Walking speed, Older adults, Younger adults, Vertical ground, reaction force
Cltation: Rastegar M, Hoseini SH, Naser Melli MS, Taffah M. [The Effects of Age and Walking Speeds on Vertical Ground Reaction Force in Younger and Older Adults (Persian)]. Journal of Rehabilitation. 2017; 17(4):290-299. http://dx.doi.org/ 10.21859 /jrehab-1704290

http://dx.doi.org/ 10.21859/jrehab-1704290

\section{ABSTRACT}

Objective Walking is one of the most important activities of daily living that plays a vital role in the lives of the elderly population and can help improve their physical and mental health. Especially, the survey of ground reaction force (GRF) during walking with different speeds between young and old adults results in better perception of essential mechanisms of speed regulation during walking. Thus, the purpose of this study was to examine the effects of age and walking speeds on vertical GRF in younger and older adults.

Materials \& Methods The subjects of this study included 15 younger and 15 older men with a mean age of 26.46 and 70.33 years, respectively. All the subjects were healthy and randomly selected. A-10-meters walking path was considered along the laboratory, and a three-axis Kistler Force plate was placed in the middle of the track. The distance of walking start line to force plate was 4 meters. Each subject walked this 10 -meters path with self-selected (100\%), slow (80\%) and fast (120\%) speeds. The vertical GRF during walking stance phases including weight acceptance or first peak phase (FZ1), midstance or depth phase (FZ2), and heel off or second peak phase (FZ3) were measured by the Force plate at 200- $\mathrm{Hz}$ frequency. The data were analyzed by ANOVA with repeated measures and Bonferroni tests.

Results The results showed that the effects of age $(P=0.002)$ and speed $(P=0.001)$ on the mean vertical GRF are significant. Also, the interaction effect of age and speed on the mean vertical GRF was significant $(P=0.013)$. According to the Bonferroni test, in both age groups, the mean vertical GRF was significantly different between different speeds $(P<0.01)$. Moreover, at $100 \%(P=0.036)$ and $120 \%(P=0.002)$ speeds, there were significant differences between younger and older adults. At slow speed, there was no significant difference in GRF components (FZ1, FZ2, and FZ3) between the two age groups (P>0.05). At self-selected and fast speeds, there were significant differences in FZ1 and FZ3 between the two groups $(P<0.01)$ while there was no significant difference regarding $F Z 2(P>0.05)$. In addition, there were significant differences at FZ1 and FZ3 between different speeds $(P<0.01)$.

Conclusion In summary, the results of the present study showed that during walking with self-selected and fast speeds, the reaction forces of weight acceptance and heel off phases were significantly different between younger and older adults, but there was no significant difference in the reaction force of midstance phase. Moreover, in both age groups, there were significant differences among walking different speeds in the reaction forces of weight acceptance and heel off phases. It seems that lower extremity muscles strengthening in the older adults can increase walking speed and decrease falling risks and functional disabilities of these people.

* Corresponding Author:
Seyyed Hosein Hoseini, PhD

Address: Department of Physical Education and Sport Sciences, Faculty of Physical Education, University of Guilan, Rasht, Iran.

Tel: +98 (919) 9196275

E-Mail: hoseini.papers@gmail.com 


\title{
بررسى اثرات سن و سرعت راهرفتن بر نيروى عكس العمل عمودى زمين در جوانان و سالمندان
}

\author{
مريم رستكار'، "سيدحسين حسينى"، محمدحسين ناصرملّى'، مرتضى تفاح' \\ 1 - كروه تربيتبدنى و علوم ورزشى، دانشكله تربيتبلنى و علوم ورزشى، واحد كرج، دانشكاه آزاد اسلامى، كرجه ايران.

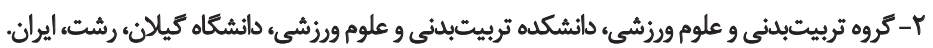

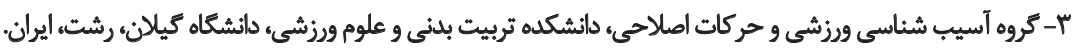

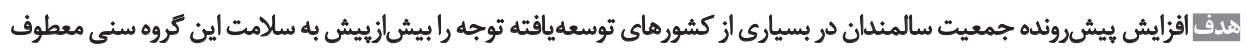

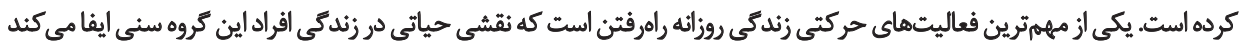

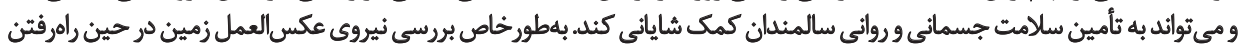

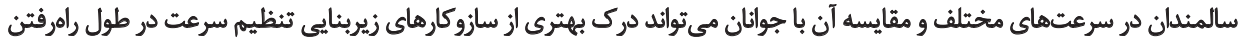

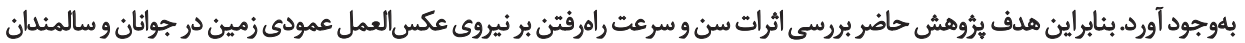

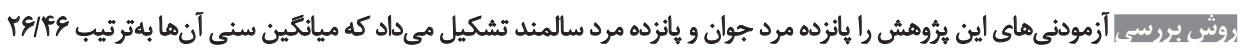

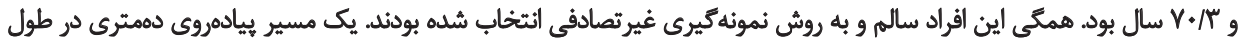

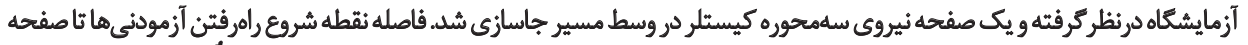

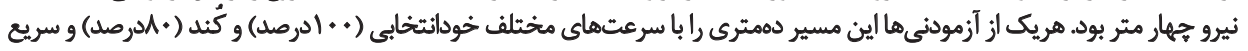

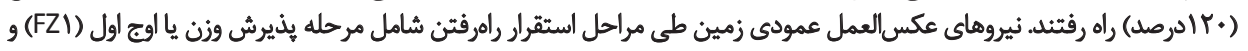

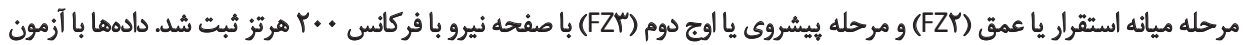

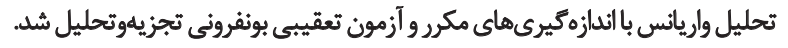

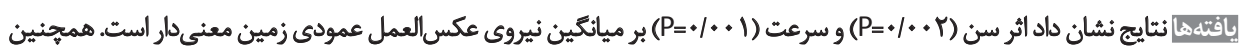

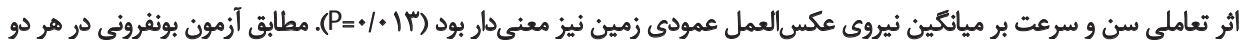

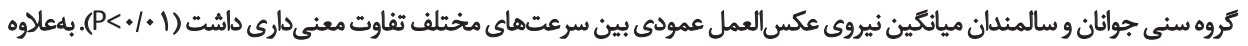

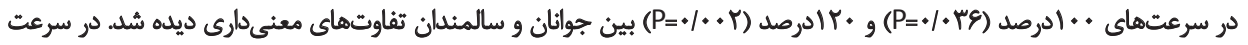

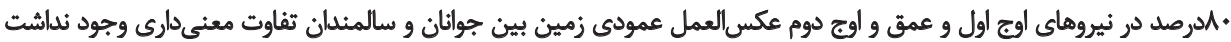

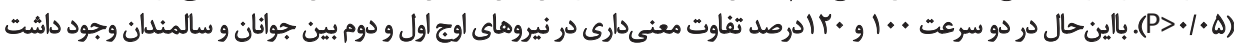

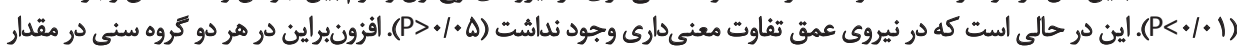

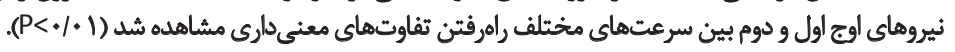

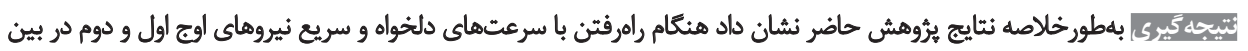

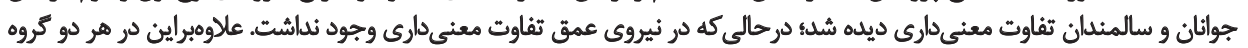

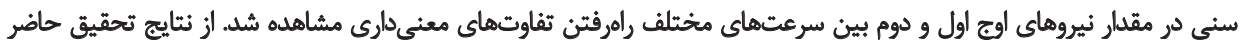

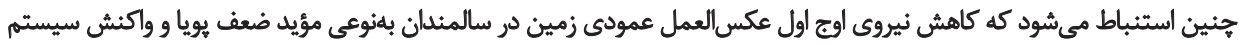

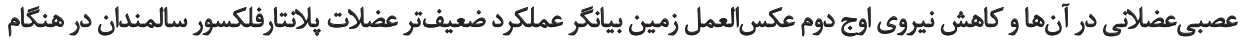

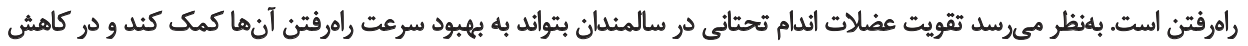

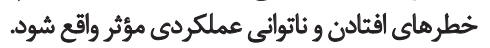

كليدوازوها:

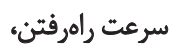

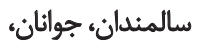
نيروى عكس العمل عمودى زمين 


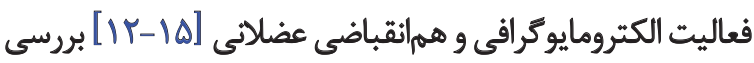

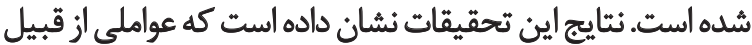

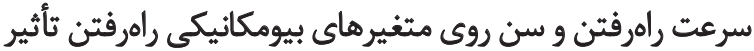

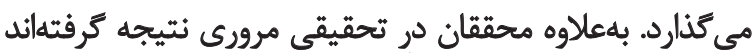

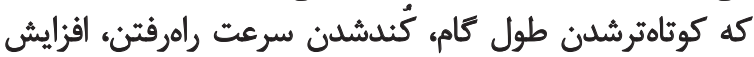

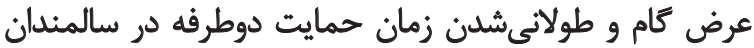

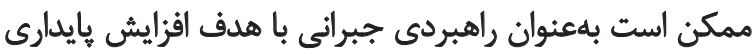

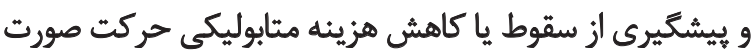

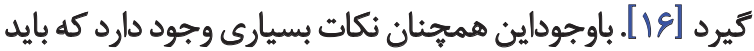
تحقيقات بيشتر و عميقترى درباره آنها انجام شودان نيروى عكس العمل زمين يكى از متغيرهاى بيومكانيكى محسوب

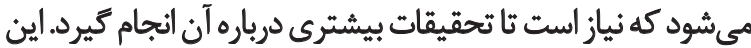

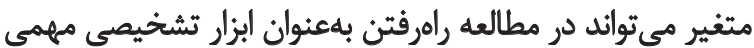

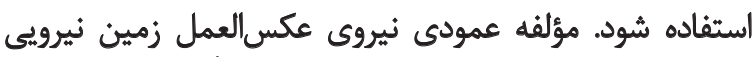

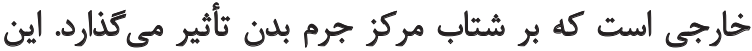

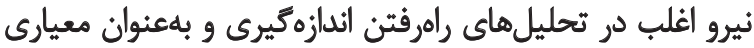

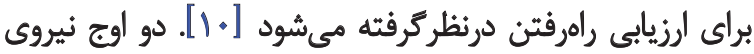

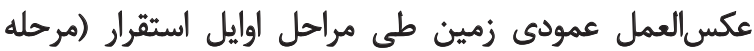

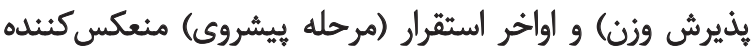

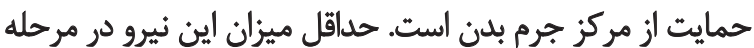

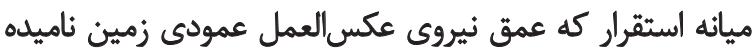

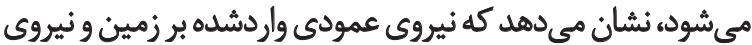

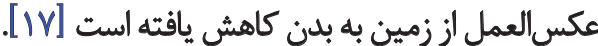
بهدليل ارتباط نيروى عكسالعمل زمين با شتاب حركت و

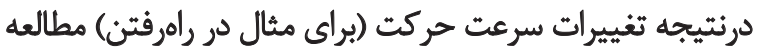

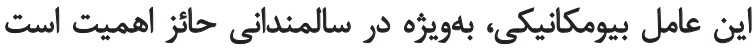

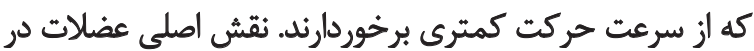

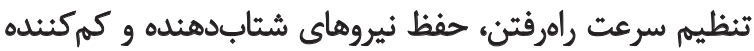

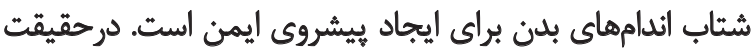

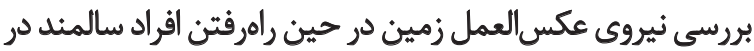

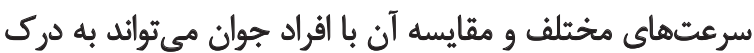

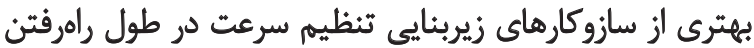

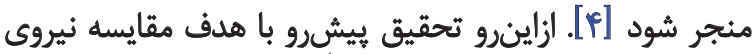

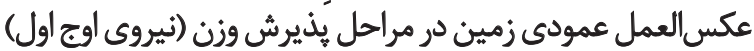

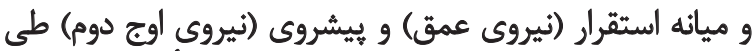

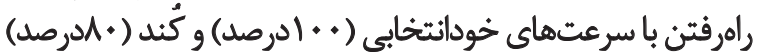

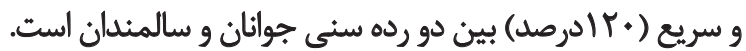

روشُ بررسى

أزمودئىها

تحقيق حاضر از نظر شيوه يروهشى از نوع نيمهنجربى و از

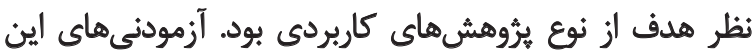

مقدمه

افزايش بيشرونده جمعيت سالمندان در بسيارى از كشورهاى

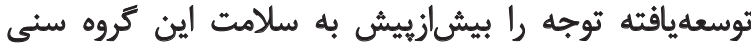

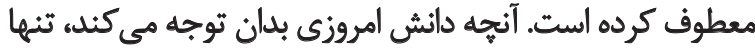

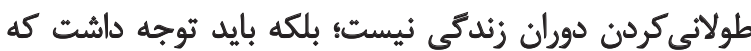

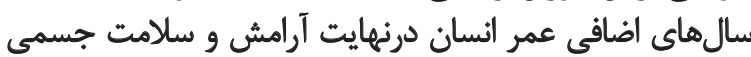

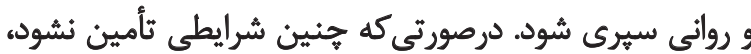

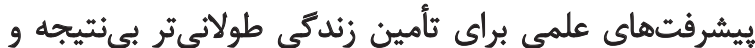

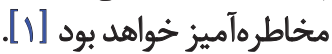

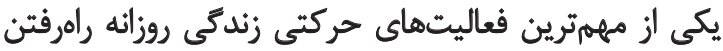

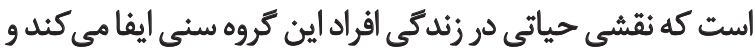

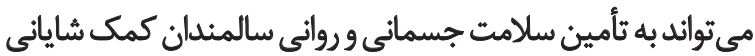

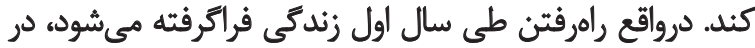

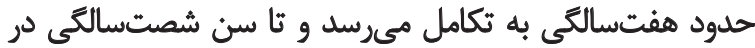

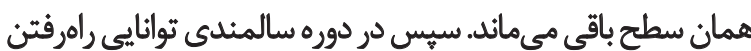

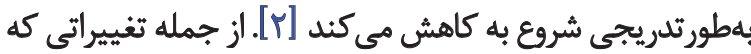

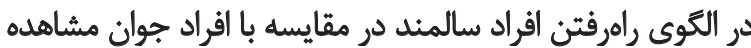

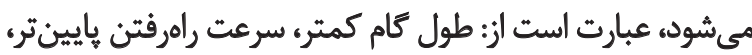

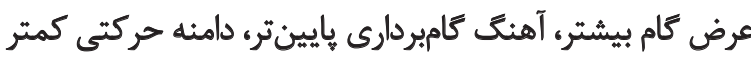

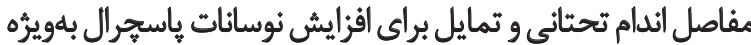

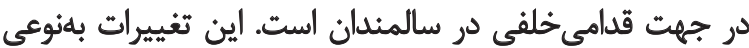

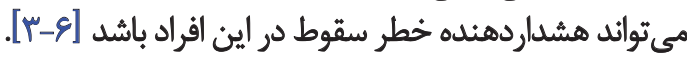
همانكونهكه بيان شد، يكى از تغييرات ايجادشده در

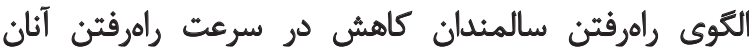

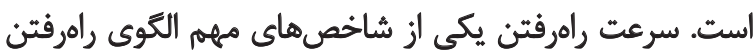

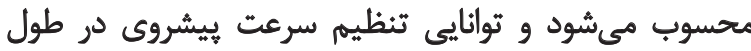

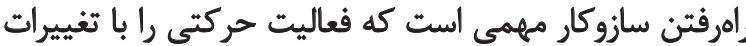

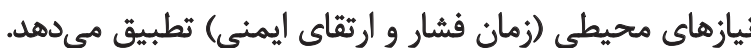

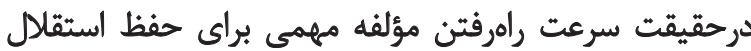

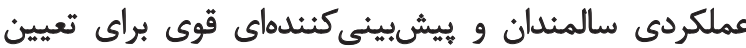

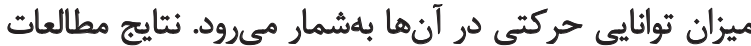

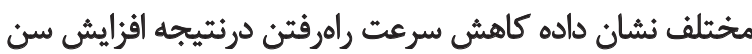
دليل تغييرات مطالبات وظايف حركتى است كه درني درنهايت منجر

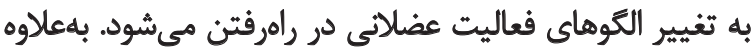

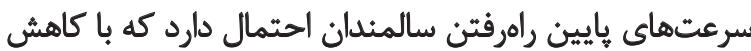

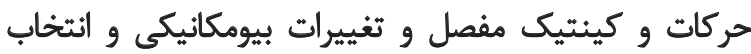

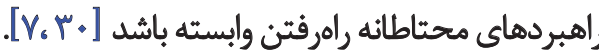

تغييرات ايجادشده در راهرفتن سالمندان در سال هاى اخير از

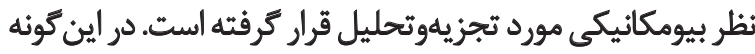

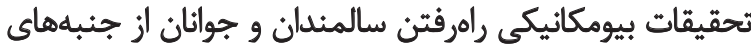

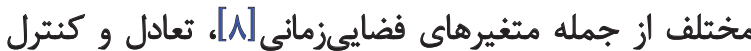

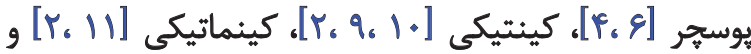


صفحه نيروسنج حين راهرفتن و قرارئقرفتن كامل گيا روى صفحه

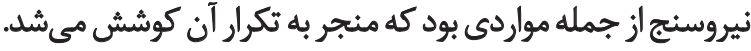

$$
\text { استخراج و محاسبه دادههاى مريوط به ئيروى عكس العمل }
$$

سن و سرعت راهرفتن بهعنوان متغيرهاى مستقل و مقدار

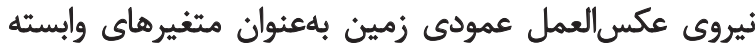

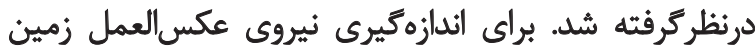

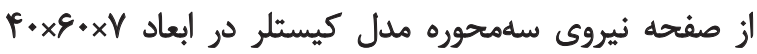

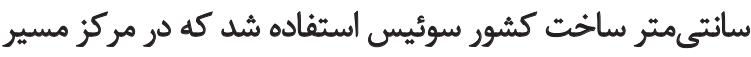

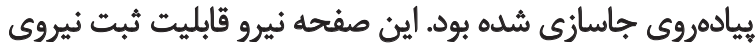
عكس العمل زمين در دامنه ده تا يانصد هرتز ران ادارد.

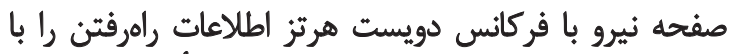

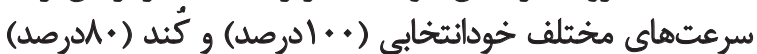

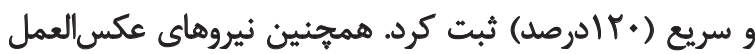

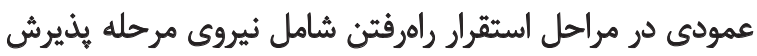

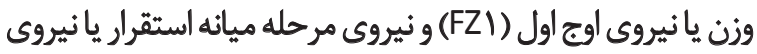

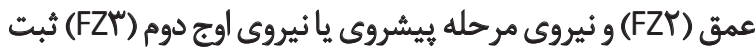

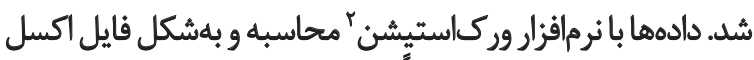

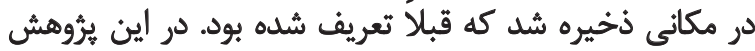

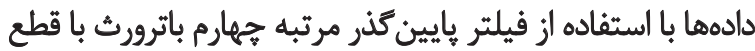

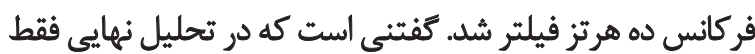
از دادههاى مربوط به مر مله استقرار استفاده شد.

$$
\text { روش هاي تحليل أمارى }
$$

بامنظور تجزيهوتحليل اطلاعات مدنظر از نسخه

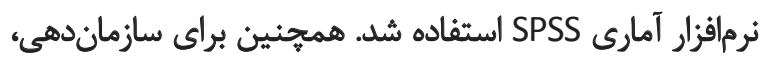

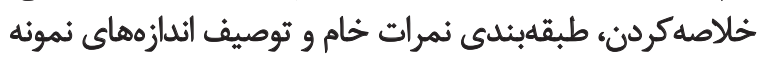

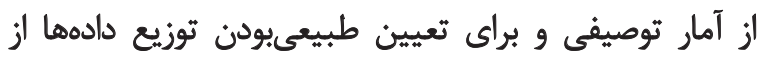

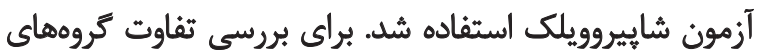

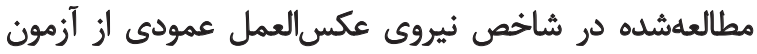

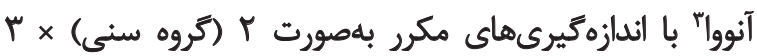

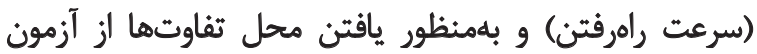

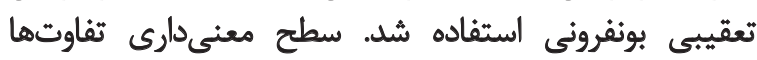

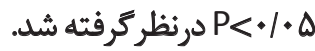

يافتهها

مقادير ميانكين و انحرافمعيار متغير سرعت راهرفتن در جدول

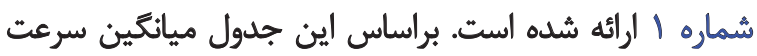

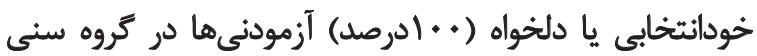

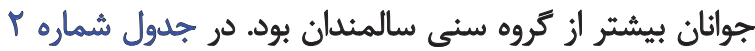

2. Workstation

3. ANOVA

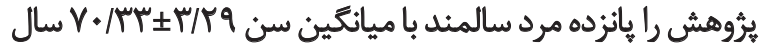

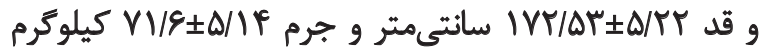

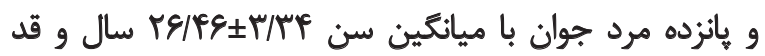

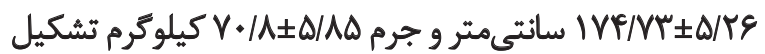

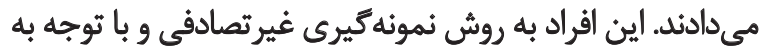
معيارهاي ورودوخروج بهصورت هدفمند انتخاب شيد شيند.

معيارهاى ورود به برثوهش عبارت بود ازئ مذكربودن، امضاى

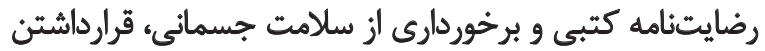

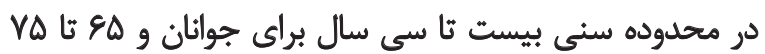

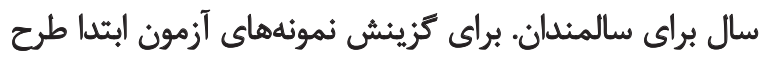

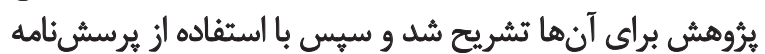

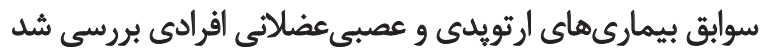

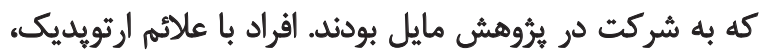

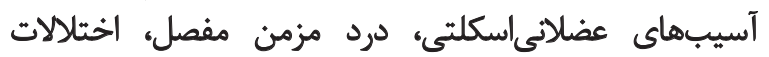

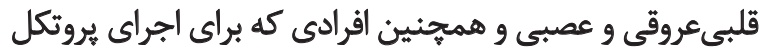

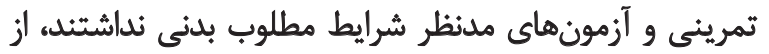

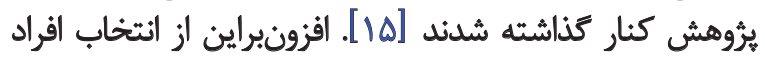

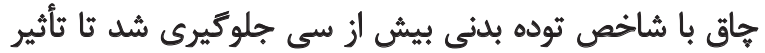

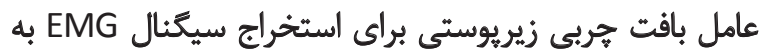

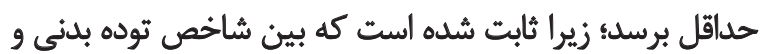

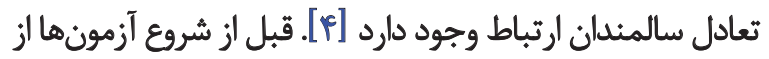

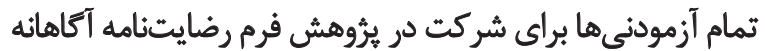

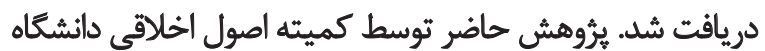

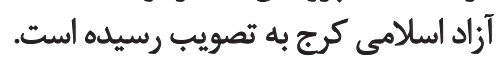

روئ اجرا يك مسير يُيادهروى دهمترى در طول آزمايشَّاه درنظركرفته

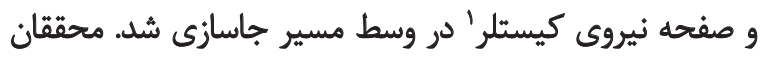

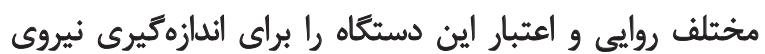

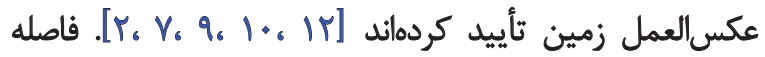

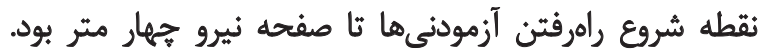

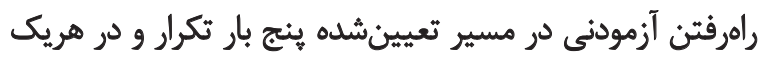

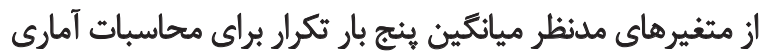

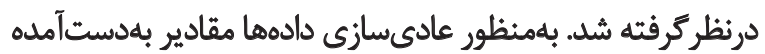

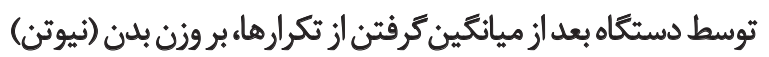

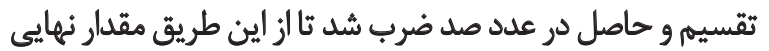

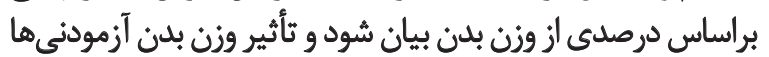

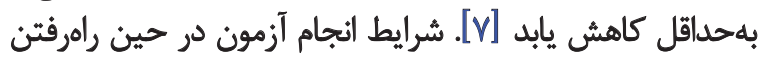

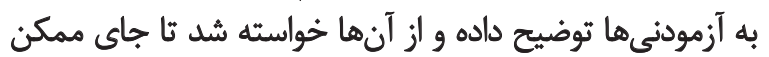

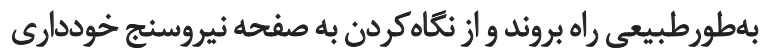

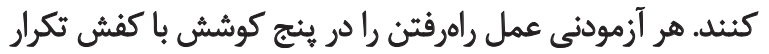

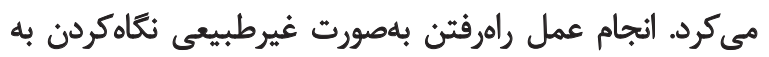


جدول ا. ميانكين و انحرافمعيار سرعت راهرفتن آزمودنىها برحسب متر بر ثائيه.

\begin{tabular}{|c|c|c|}
\hline \multicolumn{2}{|c|}{ سرعت راهرفتن (m/s) } & \multirow{2}{*}{ متغير } \\
\hline سالمندان & جوانان & \\
\hline $1 / \cdot 9 \pm+11$ & $1 / M \pm+/ 11$ & سرعت \% A \\
\hline$/ / F \varepsilon_{ \pm} / / F$ & VReAะ•/IV & سرعت \% 1. 1 \\
\hline VRru $\pm / / V$ & $1 / W \pm=19$ & سرعت \%+1F \\
\hline
\end{tabular}

توانبخننى

شد (جدول شماره س). مطابق اين جدول در هر دو كروه سنى

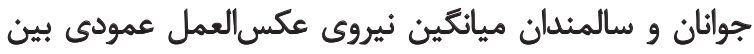

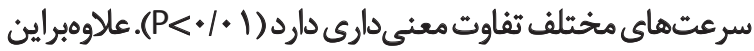

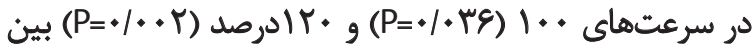
جوانان و سالمندان تفاوتهاى معنى دارى وجود دارد. براى ارائه تصويرى شماتيك از الكوى نيروى عكسالعمل

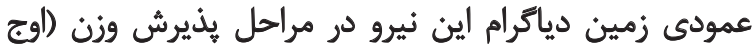

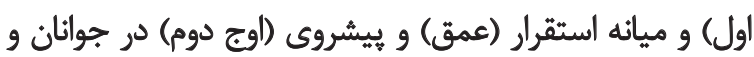

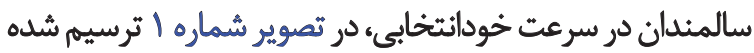

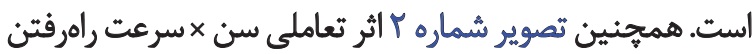

نتايج مقايسه ميانكين نيروى عكس العمل عمودى زمين (ميانكّين

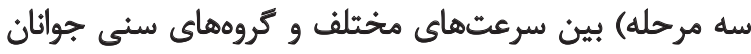

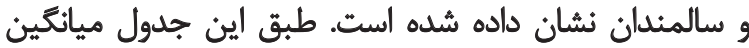

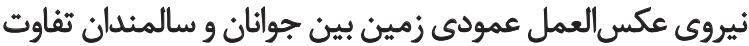

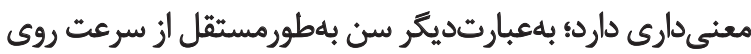

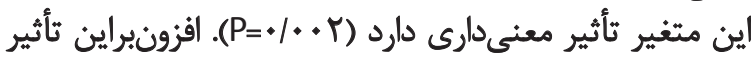

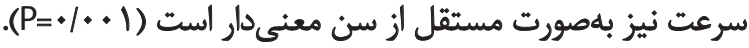

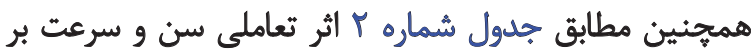

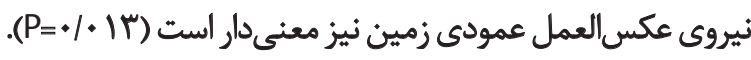
بلمنظور مقايسههاي جفتى از آزمون تعقيبى بونفرونى استفاده

جدول r. نتايج تحليل واريانس دوراهه با اندازهيرى مكرر براي مقايسه ميانكين نيروى عكس العمل عمودى زمين.

\begin{tabular}{|c|c|c|c|c|c|}
\hline مقدار P P & $F$ & مياتكين مجذورات & درجه أزادى & مجموع مجذورات & منبع تغيير \\
\hline $.0 . r$ & $w / m e$ & EIAT/AD & 1 & SIAT/AS & سن \\
\hline $.1 . .1$ & $r \Delta / r \alpha$ & $\mathrm{Rqr} / \mathrm{Ar}$ & r & Ma/8s & سرعت راهرفتن \\
\hline $.1 \%$ & $18 / 9 \mid$ & TrOVE & r & PVIVTA & سن × سرعت \\
\hline
\end{tabular}

جدول "ّ. نتايج آزمون بونفرونى براى مقايسه ميانكين نيروى عكسالعمل عمودى زمين بين جفت كروهها.

\begin{tabular}{|c|c|c|c|c|}
\hline سطح معنى داري a & خطاى انحرافـمعيار & اختالات ميانكين (J-I) & (J) & كروه (I) \\
\hline $.1+1$ & .1 .181 & $-1 / 1 \Delta \Delta$ & جواثان (\% + +1) & \\
\hline.$/ .0 \%$ & 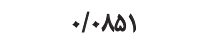 & $-r / Q V I Y$ & جواثان (\% \% & جواثان (A) \\
\hline . /1eq & .1 .101 & $-\pi /$ rit & سالمندان (A•\%) & \\
\hline $.1 .+9$ & $* 1+101$ & $-1 / 4|x\rangle$ & 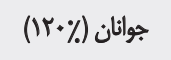 & $(1) .4$ \\
\hline ( & .1 .181 &.$/ 4 r$ & سالمثلدان (\% +.1) & \\
\hline$+1++Y$ & $* 1+181$ & V/FVA & سالمندان (\% + Ir) & جوانان (Ir + \\
\hline $.1+5$ & $.1+181$ & - & سالمندان (\% + 1) & \\
\hline $.1 . .1$ & $.1 \cdot N \Delta 1$ & -1/qATT & سالمثدان (1r\%) & Uالهمان (A+\%) \\
\hline 1.4 & $\%+101$ & $-* / V \wedge 9$ & سالمثدان (1T\%) & سالمندان (1.0\%) \\
\hline
\end{tabular}




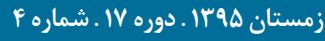

بررسى اثرهاى سن و سرعت راهرفتن بر نيروى عكس العمل عمودى زمين در جوانان و سالمندان

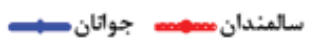

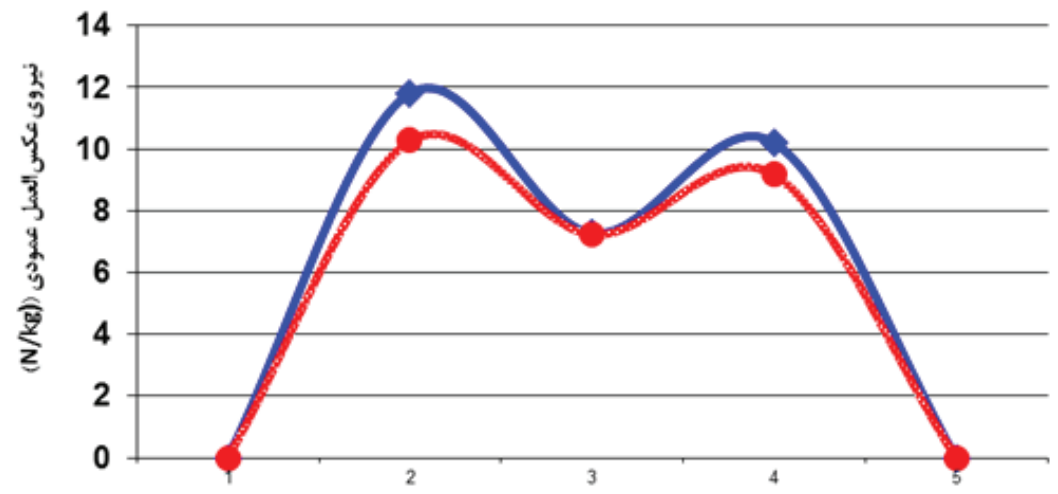

توانبخنتى

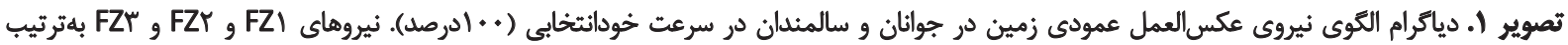

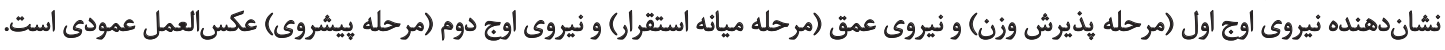

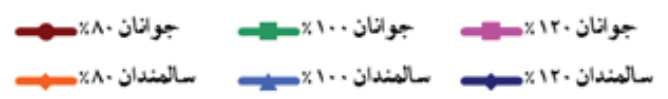

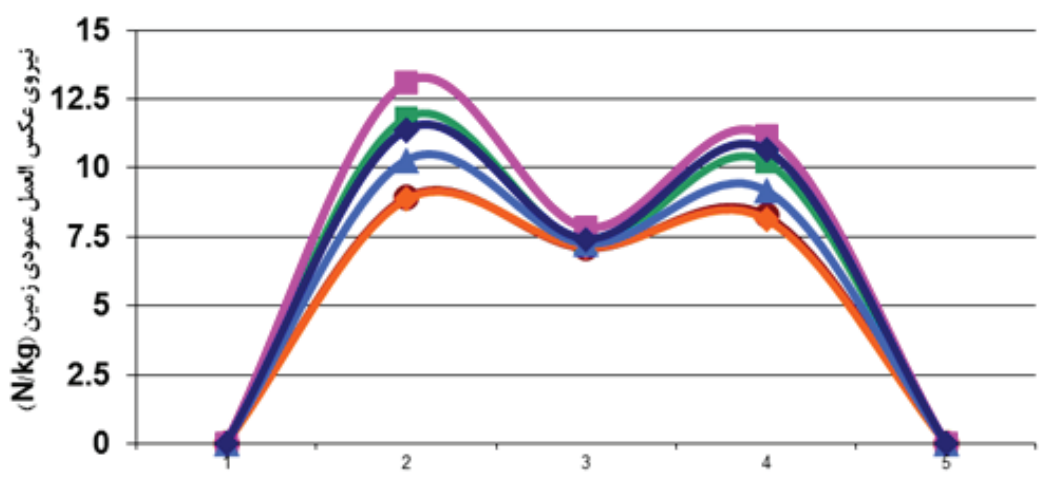

توانبخننى

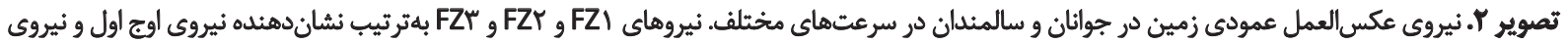
عمق و نيروى اوج دوم عكس العمل عمودى است عمدئ.

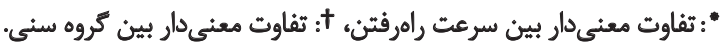

زمين طى راهرفتن با سرعتهاى خودانتخابى (· (. ادرصد) و كُند

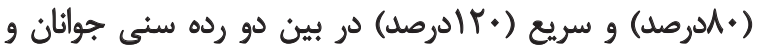

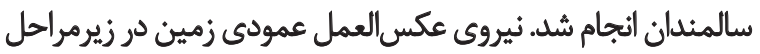

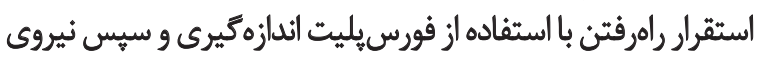
عكس العمل عمودى براساس جرم بدن طبيعى شد.

براساس نتايج يُؤوشب حاضر در ميانغًين نيروى عكسالعمل

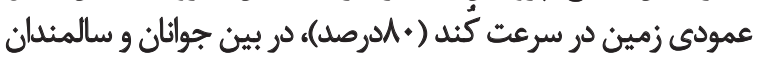

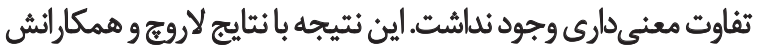

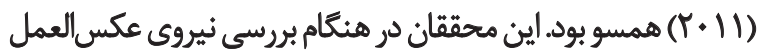

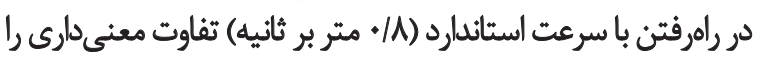

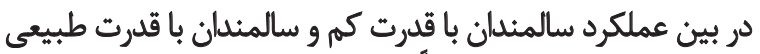

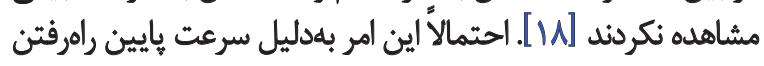

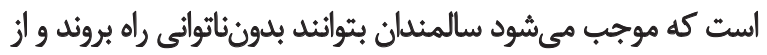

را روى تغييرات نيروى عكس العمل عمودى زمين نشان مى دهد.

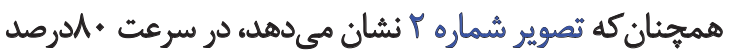

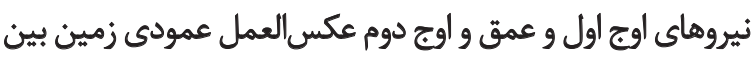

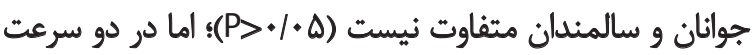

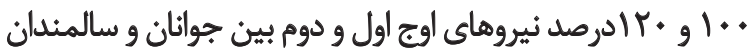

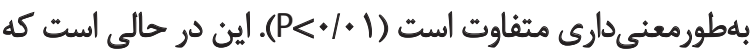

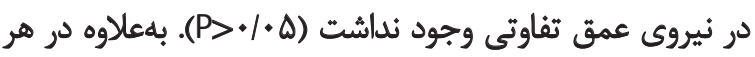

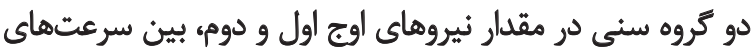

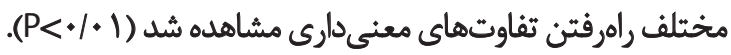

$\stackrel{\leftrightarrow}{\leftrightarrow}$

ثروّهش حاضر با هدف مقايسه نيروى عكسالعمل عمودى

rqd 


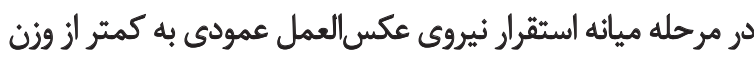

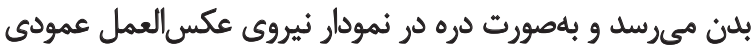

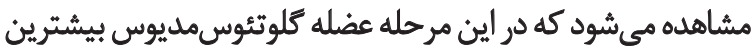
حمايت را فراهم مي كند.

در مرحله انتهايي استقرار كه در آن شتاب بلدن درخلاف شتاب

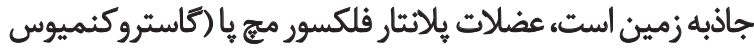

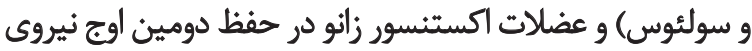

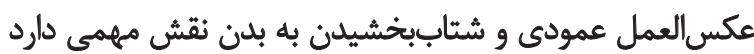

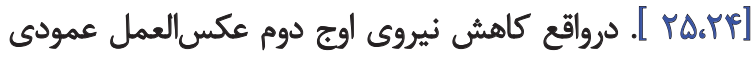

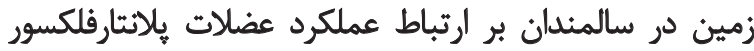

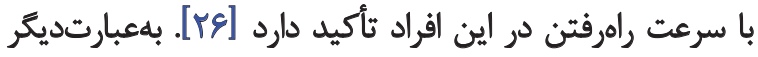

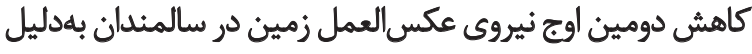

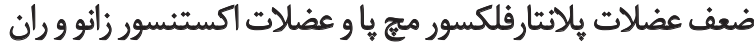

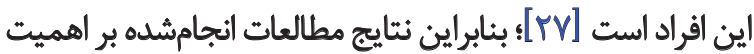
تقويت اين عضلات در سالمندان نأكيد دارد.

براساس نتايج يزوهش حاضر ميانكّين نيروى عكس العمل عمودى

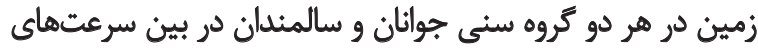

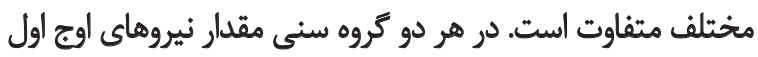

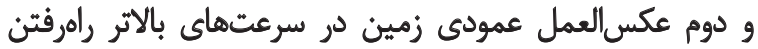

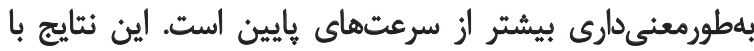

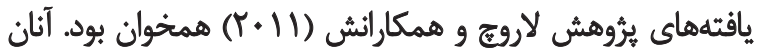

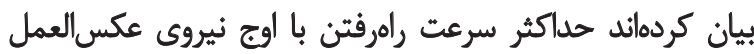

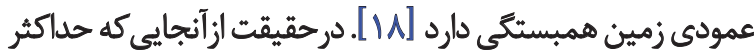

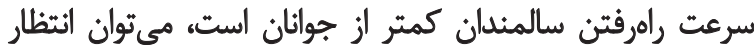

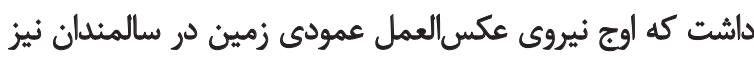

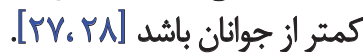

بهدليل وجود رابطه خطى مثبت در بين اوج نيروى عكسالعمل

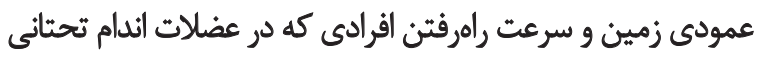

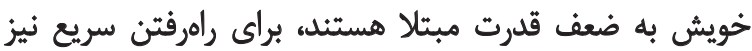

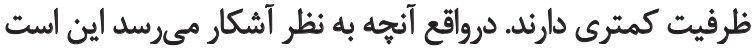

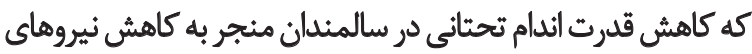

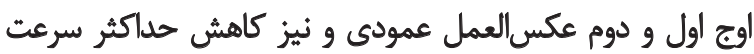

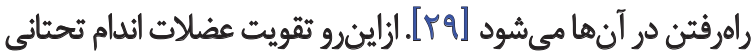

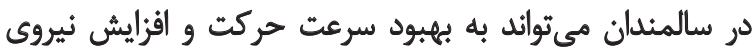

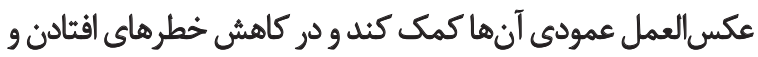

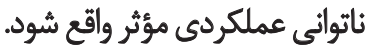

\section{نتيجلكَيرى}

براساس نتايج يروهش حاضر ميانكين نيروى عكسالعمل

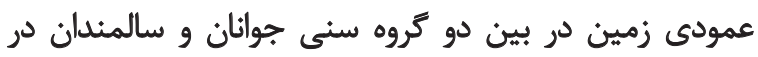

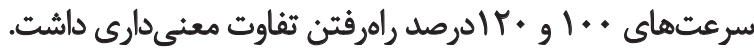

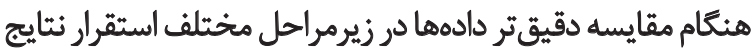

عملكردى مشابه باگروه جوانان برخوردار باشند [. ، Y [1]]

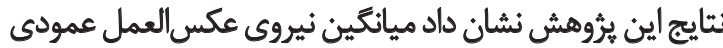

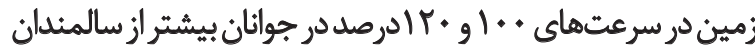

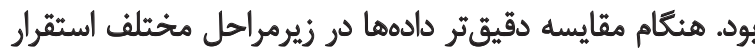

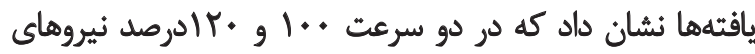

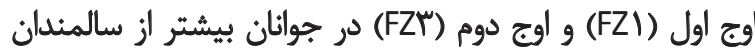

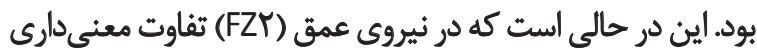

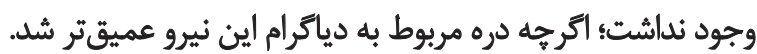

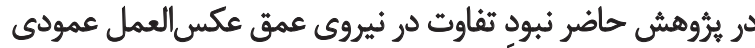

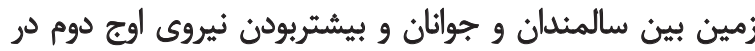

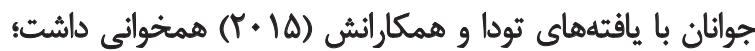

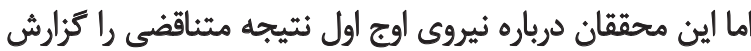

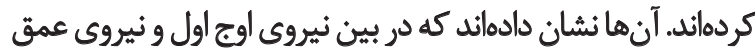

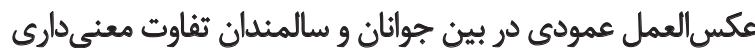

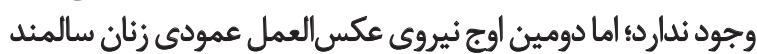

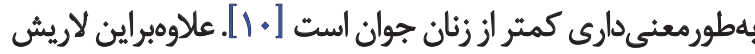

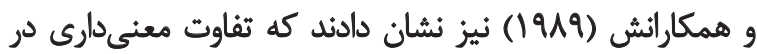
FZI

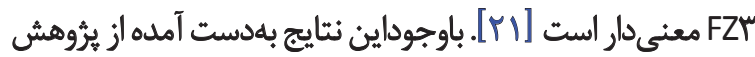

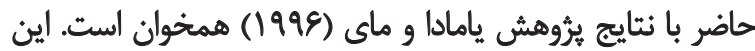

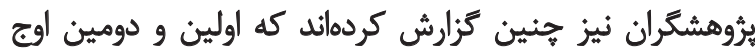

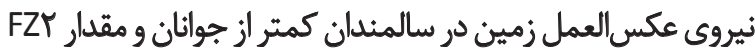

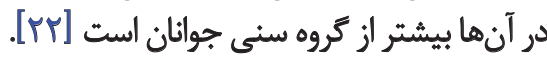
ازآنجايى كه مقدار اولين و دومين اوج نيروى عكسالعمل زمين

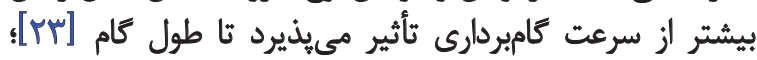

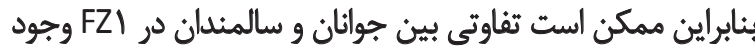

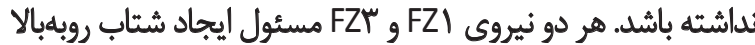

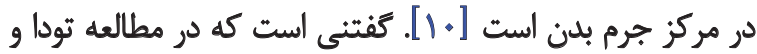

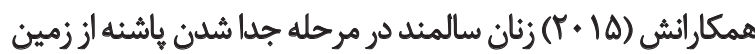

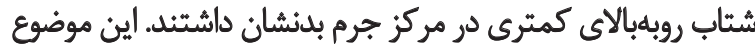

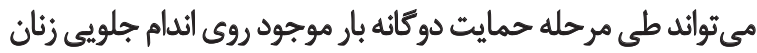

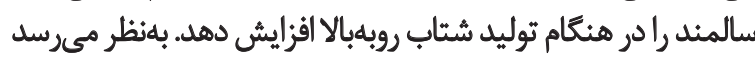

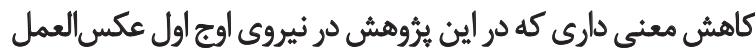

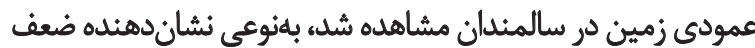

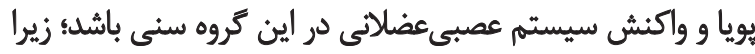

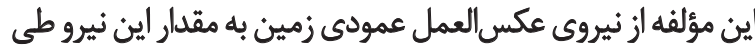

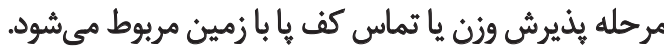

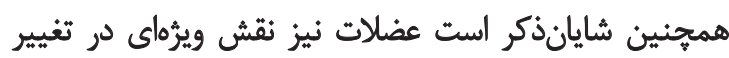

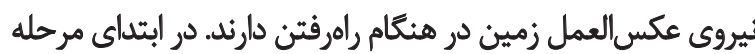

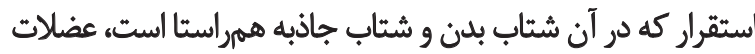

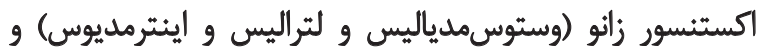

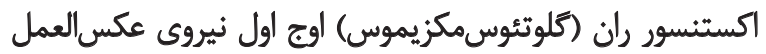

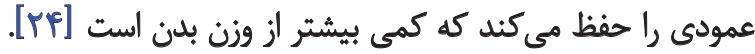




\section{References}

[1] Shoaee F, Azkhosh M, Alizad V. [Health status of Iranian older people: A demographical Analysis (Persian)]. Iranian Journal of Ageing. 2013; 8(2):60-69.

[2] Paroczai R, Bejek Z, Illyés A. Kinamatic and kinetic parameters of healthy elderly people. Periodica Polytechninca Ser Mechanical Engineering. 2005; 49(1): 63-67.

[3] Akbari-Kamrani AA, Azadi F, Salavati M, Kazemi B. [Predicting fall risk in nursing home elderly using two functional assessment methods (Persian)]. Journal of Rehabilitation. 2003; 4(2):45-52.

[4] Baharlouei H, Nodehi-Moghaddam A. [Correlation between Body Mass Index and Postural Balance in Elderly (Persian)]. Journal of Rehabilitation. 2012; 12:54-9.

[5] Kimura T, Kobayashi H, Nakayama E, Hanaoka M. Effects of aging on gait patterns in the healthy elderly. Anthropological Science. 2007; 115(1):67-72. doi: 10.1537/ase.060309

[6] Gomes MM, Reis JG, Neves, TM, Petrella, M, Abreu DC. Impact of aging on balance and pattern of muscle activation in elderly women from different age groups. International Journal of Gerontology. 2013; 7(2):106-11. doi: 10.1016/j.ijge.2012.11.013

[7] Winter DA. The biomechanics and motor control of human gait: normal, elderly and pathological $2^{\text {nd }}$ ed. Ontario: University of Waterloo Press; 1991.

[8] Sadeghi H, Norouzi H. [Spatio-Temporal Parameters Changes in Gait of Male Elderly Subjects (Persian)]. Iranian Journal of Ageing. 2010; 4(2):71-76.

[9] Toda H, Nagano A, Luo Z. Contribution of lower extremity joint moment on ground reaction force during walking in elderly and younger people.Paper presented at: 2013 IEEE/SICE International Symposium on System Integration; 2013 Dec 15-17; Kobe, Japan.

[10] Toda H, Nagano A, Luo Z. Age and gender differences in the control of vertical ground reaction force by the hip, knee and ankle joints. Journal of Physical Theraphy Science. 2015; 27(6):1833-838. doi: 10.1589/jpts.27.1833

[11] Arshi AR, Eskandari F, Navvab Motlagh F, Karimi M. [ComparE ing pelvic and trunk kinematics between young and older adults during treadmill walking (Persian)]. Paper presented at: $21^{\text {st }}$ Iranian Conference on Biomedical Engineering; 26-28 Nov 2014; Tehran, Iran.

[12] Larsen AH, Puggaard L, Hamalainen U, Aagaard P. Comparison of ground reaction forces and antagonist muscle coactivation during stair walking with ageing. Journal of Electromyography and Kinesiology. 2008; 18(4):568-80. doi: 10.1016/j. jelekin.2006.12.008

[13] Hortobagyi T, Solnik S, Gruber A, Rider P, Helseth J, DeVita P. Interaction between age and gait velocity in the amplitude and timing of antagonist muscle co-activation. Gait \& Posture. 2009; 29(4): 558-64. doi: 10.1016/j.gaitpost.2008.12.007

[14] Schmitz A, Silder A, Heiderscheit B, Mahoney J, Thelen DG. Differences in lower-extremity muscular activation during walking between healthy older and young adults. Journal of Electromyo-
نشان داد كه در اين سرعتها نيروهاي اوج اول و دوم در بين

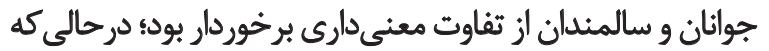

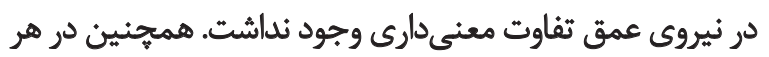

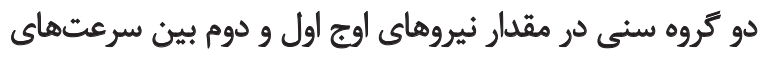

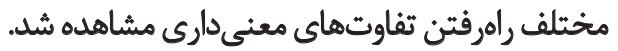

بلهور كلى از نتايج تحقيق حاضر جنين استنباط ميشود كه

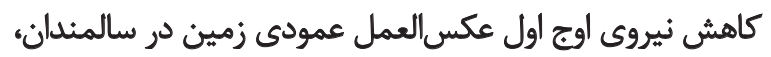
بلنوعى مؤيد ضعف يويا و واكنش سيستم عصبى عضلانى در آنه آنها

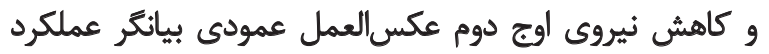

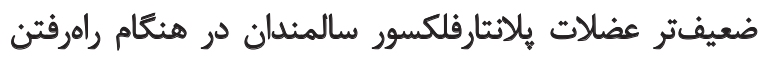
است. بهنظر مىرسد تقويت عضلات اندام تحتانى در سالمئدان

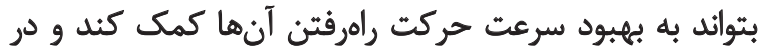
كاهش خطر هاي افتادن و ناثوائي عملكردى مؤثر واقع شود. برد

مهندويت

ازجمله محدوديتهاي يُروهش حاضر ميسرنبودن يكسانسازى

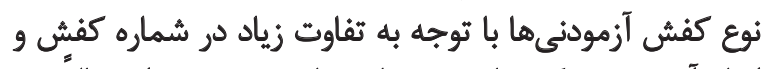

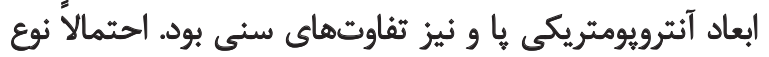
كفش بتواند بر ميزان نيروهاى عكس العمل زئين زمين مؤثر باشد. Losinging

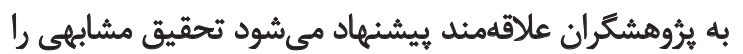

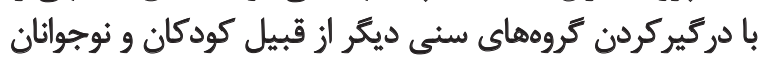

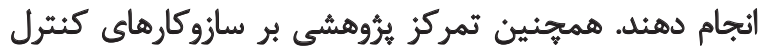

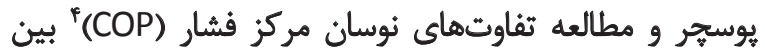

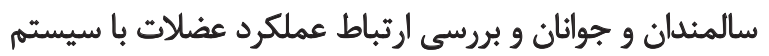

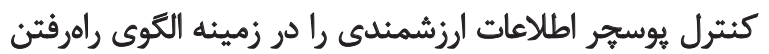

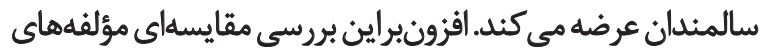

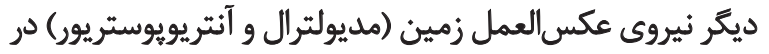

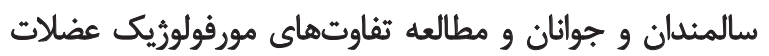

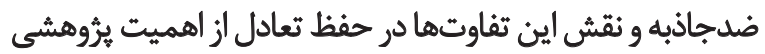
زيادى برخوردار است.

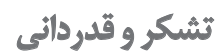

اين مقاله بركرفته از يايان نامه كارشناسي ارشد نويسنده اول

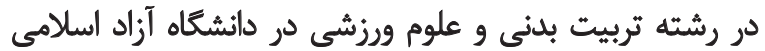
واحد كرج است. 
graphy and Kinesiology. 2009; 19(6):1085-091. doi: 10.1016/j. jelekin. 2008.10 .008

[15] Khodaveisi H, Anbarian M, Khodaveisi M. [Comparison of Electromyographic Activity Pattern of Knee Two-Joint Muscles between Youngs and Olders in Gait Different Speeds (Persian)]. Journal of Rehabilitation. 2015; 16(4): 234-41.

[16] Aboutorabi A, Arazpour M, Bahramizadeh M, Hutchins SW, Fadayevatan R. The effect of aging on gait parameters in ablebodied older subjects: a literature review. Aging Clinical and Experimental Research. 2016; 28(3):393-405. doi: 10.1007/s40520015-0420-6

[17] Lin MR, Hwang HF, Wang YW, Chang SH, Wolf SL. CommuniH ty-Based Tai Chi and its effect on injurious falls, balance, gait and fear of falling in older people. Physical Therapy. 2006; 85(9):1189201.doi: 10.2522/pti.20040408

[18] LaRoche DP, Millett ED, Kralian RJ. Low strength is related to diminished ground reaction forces and walking performance in older women. Gait \& Posture. 2011; 33(4):668-72. doi: 10.1016/j. gaitpost.2011.02.022

[19] Lauretani F, Russo CR, Bandinelli S, Bartali B, Cavazzini C. Age-associated changes in skeletal muscles and their effect on mobility: an operational diagnosis of sarcopenia. Journal of Applied Physiology. 2006; 95(5):1851-60. doi: 10.1152/japplphysiol.00246.2003

[20] Bouchard DR, Janssen I. Dynapenic-obesity and physical function in older adults. Journals of Gerontology Series A: Biological Sciences and Medical Sciences. 2011; 65(1):71-7. doi: 10.1093/ gerona/glp159

[21] Larish DD, Martin PE, Mungiole M. Characteristic patterns of gait in the healthy old. Annals of the New York Academy of Sciences. 1989; 515(1):18-32. doi: 10.1111/j.1749-6632.1988. tb32960.x

[22] Yamada T, Maie K. The characteristics of walking in old men analyzed from the ground reaction force. Journal of Anthropological Society of Nippon. 1996; 96(1):7-15. doi: 10.1537/ ase1911.96.7

[23] Martin PE, Marsh AP. Step length and frequency effects on ground reaction forces during walking. Journal of Biomechanics. 1993; 25(10):1237-1239. doi: 10.1016/0021-9290(92)90081-b

[24] Liu MQ, Anderson FC, Pandy MG, Delp SL. Muscles that supF port the body also modulate forward progression during walking. Journal of Biomechanics. 2006; 39(14):2623-630. doi: 10.1016/j. jbiomech.2005.08.017

[25] Kepple TM, Siegel KL, Stanhope SJ. Relative contributions of the lower extremity joint moments to forward progression and support during gait. Gait \& Posture. 1997; 6(1):1-8. doi: 10.1016/ s0966-6362(96)01094-6

[26] Keller TS, Weisberger AM, Ray JL, Hasan SS, Shiavi RG, Spent gler DM. Relationship between vertical ground reaction force and speed during walking, slow jogging, and running. Clinical Biomechanics. 1999; 11(5):253-59. doi: 10.1016/0268-0033(95)00068-2

[27] McGibbon CA, Krebs DE. Discriminating age and disability effects in locomotion: neuromuscular adaptations in musculoskele- tal pathology. Journal of Applied Physiology. 2008; 96(1):149-60. doi: 10.1152/japplphysiol.00422.2003

[28] Devita P, Hortobagyi T. Age causes a redistribution of joint torques and powers during gait. Journal of Applied Physiology. 2007; 88(5):1804-811. PMID: 10797145

[29] Rantanen T, Guralnik JM, Izmirlian G, Williamson JD, Simonsick EM, Ferrucci L. Association of muscle strength with maximum walking speed in disabled older women. American Journal of Physical Medicine \& Rehabilitation. 1988; 77(4):299-305. doi: 10.1097/00002060-199807000-00008

[30] Akbari Kamrani AA, Zamani Sani SH, Fathi Rezaie Z, Aghdasi MT. Concurrent validity of functional gait assessment, timed up and go, and gait speed tests in the Persian community-dwelling elderly. Iranian Rehabilitation Journal. 2010; 8(2):15-20. 
